\title{
Емкостной дилатометр для измерения магнитострикции, пьезоэлектрического эффекта и коэффициента линейного температурного расширения
}

\author{
Фрейдман А.Л. ${ }^{1}$, Попков С.И. ${ }^{1}$, Семенов С.В. ${ }^{1}$, Турчин П.П. ${ }^{2}$ \\ 1 - Институт физики им. Л.В. Киренского, ФИЦ КНЦ СО РАН, \\ 660036, г. Красноярск, Россия \\ 2 - Сибирский федеральный университет, 660041, г. Красноярск, Россия \\ fss4@yandex.ru
}

\begin{abstract}
Разработан емкостной дилатометр для измерений магнитострикции, пьезоэлектрического эффекта и коэффициента линейного теплового расширения в диапазоне температур от 1.85 до 350 К, во внешнем магнитном поле вплоть до 90 кЭ И внешнем электрическом поле с напряжением до 1 кВ, работающего на базе коммерческой установки по исследованию свойств твердых тел PPMS QD.
\end{abstract}

Деформация вещества, вызванная изменением внешних условий, отражает многие процессы, происходящие на уровне элементарной ячейки и межатомных взаимодействий. Так, например, при фазовых переходах моно- и поликристаллических соединений сложно установить процессы, происходящие внутри элементарной ячейки прямыми методами (рентгеновские и нейтронографические измерения), однако результатом этих преобразований является общая деформация образца, которую можно зафиксировать методами дилатометрии непосредственно в точке фазового перехода и ее окрестностях. По этой причине список публикаций по теме разработок в области измерений малых деформаций пополняется и по настоящий день [1], несмотря на то, что такие методы существуют уже довольно давно.

Актуальность построения новых экспериментальных установок также обусловлена появлением новых задач перед измерительной техникой. Примером этого могут служить мультиферроики, где деформация играет важную роль. Имея образец, который проявляет значительные магнитострикционные свойства и одновременно является пьезоэлектриком, возможно индуцирование электрической поляризации посредством магнитного поля. Связь магнитной подсистемы с электрическими свойствами открывает огромные 
перспективы использования мультиферроиков, начиная от датчиков магнитного поля и заканчивая новыми типами оперативной памяти компьютеров и логическими элементами [6-8].

Для исследования подобных образцов важно иметь возможность измерения не только коэффициента линейного теплового расширения или магнитострикции. Имея сложные связи между различными типами деформаций необходимы измерительные установки, которые могли бы проводить измерения в широком спектре внешних условий. Так, для мультиферроиков важно проводить измерения относительной деформации, вызванной влиянием температуры, магнитного и электрического полей. Кроме того, представляет интерес поведение относительной деформации при приложении одновременно и магнитного, и электрического полей. Таким образом, разработка таких измерительных установок является актуальной задачей, учитывая то, что публикации по этому вопросу отсутствуют в литературе.

При реализации дилатометрических измерений емкостным методом используется измерительный конденсатор, одна из обкладок которого является подвижной и изменяет свое положение под действием деформации образца относительно неподвижной обкладки. При этом изменяется емкость конденсатора, которая в случае плоского конденсатора определяется выражением

$$
C=\varepsilon \cdot \varepsilon_{0} * \frac{S}{d},(1)
$$

где $\varepsilon$ - относительная диэлектрическая проницаемость объема между обкладками, $\varepsilon_{0}-$ диэлектрическая проницаемость вакуума, $s$ - площадь обкладок конденсатора, $d$ расстояние между обкладками. Изменение емкости $\Delta C$ можно интерпретировать как изменение расстояния между обкладками $\Delta d$. Эту зависимость легко определить из (1)

$$
\Delta d=-\frac{\varepsilon \cdot \varepsilon_{0} \cdot s \cdot \Delta C}{C_{\mathbf{1}} \cdot C_{\mathbf{2}}}
$$


где $C_{1}$ - емкость конденсатора в начальный момент времени, $C_{2}$ - емкость, измеренная после деформации образца, $\Delta C=C_{2}-C_{1}$. Знак «-» в выражении (2) указывает на то, что при увеличении расстояния между обкладками емкость конденсатора уменьшается.

Деформация образца может быть вызвана различными причинами, такими как тепловое расширение, магнитострикция, пьезоэлектрический эффект, электрострикция, пьезомагнитный эффект. Для решения такого большого спектра задач нами была разработана дилатометрическая ячейка, изображенная на рисунке 1 , адаптированная для использования в измерительной системе PPMS QD.

Конструкция измерительной ячейки схожа с конструкциями, представленными в работах $[5,6]$, однако, имеются отличия. Главным отличием является наличие дополнительных токоподводящих контактов для измерения деформации образца, вызванной приложением внешнего электрического поля, другая организация гальванической развязки между подвижной обкладкой, ее подвесной пружиной и корпусом ячейки. Конструкция ячейки представлена на рисунке $1 b$.

Образец располагается на столике образца 1. Столик совмещен с корпусом измерительной ячейки 2 резьбовым соединением М5х0.5. Оптимальная высота столика (зазор между столиком и подвижной обкладкой конденсатора 3) достигается путем его вращения. Для установки точного положения столика предусмотрена передача вращения через редуктор, который зацепляется с зубчатым колесом 4. Гайка 5 служит для фиксации зубчатого колеса. После выполнения настройки высоты столика, достигнутое положение фиксируется контргайкой 6. Подвижная обкладка 3 подвешена на пружине мембранного типа 7, которая крепится с помощью гайки 8. Для исключения электрического контакта между подвижной обкладкой и корпусом измерительной ячейки предусмотрены полиимидные прокладки 9. Подвижная обкладка находится внутри кольца 10. Неподвижная обкладка 11 вклеена в кольцо неподвижной обкладки 12. Для обеспечения изначального зазора между двумя обкладками измерительного конденсатора служит 
прокладка 13. Переходная пластина 14 служит для крепления электрического разъема 15, адаптированного под электрический разъем установки PPMS QD.

Основным материалом измерительной ячейки является медь марки M1, из которой изготовлено большинство деталей. Пружина мембранного типа 7 изготовлена из беррилиевой бронзы БрБ2, неподвижная обкладка 11 вклеена в кольцо 10 при помощи криогенного теплопроводного клея Stycast 2850 FT. Зубчатое колесо 4 изготовлено из пластика.

При измерениях образец зажимается между столиком 1 и подвижной обкладкой 3 с некоторым натяжением мембраны 7, до того состояния когда емкость полученного конденсатора окажется в удобном диапазоне для измерений.

Измерительный конденсатор не может быть выполнен идеально, всегда присутствуют отклонения от закона плоского конденсатора, вызванные шероховатостью поверхности, неплоскопораллельностью обкладок конденсатора и их несоосностью. Поэтому целесообразно определить коэффициент связи между емкостью измерительного конденсатора и расстоянием между обкладками экспериментально, а также определить рабочий диапазон измерительного конденсатора.

Для проверки выполнения закона плоского конденсатора нами были проведены калибровочные измерения емкости в зависимости от смещения подвижной обкладки конденсатора. Результат измерений представлен на рисунке 2. Как видно из рисунка зависимость емкости измерительного конденсатора от смещения подвижной обкладки несколько отличается от линейного закона в верхнем и нижнем диапазонах емкостей, но хорошо согласуется в промежуточной области. Таким образом, рабочим диапазоном измерительной ячейки является диапазон изменения емкости от 13.5 до 32 пФ, что соответствует деформации образца 110 мкм. Чувствительность измерительной ячейки составляет $1.85 \cdot \AA$, однако стоит указать, что такая точность возможна лишь при больших значениях измеряемой емкости. 
При измерениях коэффициента линейного теплового расширения (КЛТР) деформации подвержен не только исследуемый образец, но и сама измерительная ячейка, так как материал ячейки также деформируется при изменении температуры, вследствие чего измеряемая емкость отражает сумму деформаций образца и ячейки. Для того чтобы определить вклад ячейки при измерениях КЛТР можно проводить измерения КЛТР известных образцов, после чего произвести расчет деформации ячейки путем вычета деформации образца.

Для калибровки измерений КЛТР мы, как и авторы других работ [5, 7], провели температурные измерения емкости измерительной ячейки с образцами чистых металлов (серебро 99.99\%, алюминий 99.995\%, медь 99.997\%), для которых известен КЛТР.

Обратный пьезоэлектрический эффект заключается в деформации образца при приложении внешнего электрического поля. Этот эффект, как правило, очень мал, поэтому мы не смогли найти работу, где были бы проведены такие измерения на емкостном дилатометре. Однако, мы зафиксировали этот эффект с помощью разработанного дилатометра. В качестве образца мы использовали монокристалл алюмобората гольмия $\mathrm{HoAl}_{3}\left(\mathrm{BO}_{3}\right)_{4}$, который проявляет гигантский магнитоэлектрический эффект и привлекает большое внимание со стороны исследователей [8-10].

Электрическое поле к кристаллу прикладывалось вдоль кристаллографического направления $a$, деформация измерялась также вдоль этого направления при температуре $T=4.2$ К. Для обеспечения однородности электрического поля, к образцу были приклеены обкладки из медной фольги размером, превосходящим размер кристалла (вставка к рисунку 3а). Между электродами и измерительной ячейкой проложены диэлектрические прокладки для исключения пробоя на измерительную ячейку.

На рисунке 3a,b представлены графики временной зависимости емкости измерительного конденсатора. Каждая ступень на графике соответствует изменению приложенного электрического поля на 100 В (кристалл имеет толщину 1 мм вдоль 
направления приложенного поля). Как видно из графика, при измерениях наблюдается экспериментальный дрейф электрической емкости (рис. 3a), который может быть связан с температурными флуктуациями. Экспериментальный дрейф можно вычесть при обработке данных (рис. 3b).

Ввиду крайней малости изменения емкости на всем диапазоне приложенных электрических полей можно интерполировать зависимость емкости конденсатора от напряженности электрического поля $C(E)$ линейной функцией (рис. 3c). На рисунке 3в представлены относительные и абсолютные значения деформации образца, которая рассчитывалась исходя из калибровочных данных (рис. 2).

Как видно из графика зафиксированное значение абсолютной деформации составило всего $~ 1.5$ нм при приложении электрического поля 6 кВ/см. Что говорит о высокой чувствительности собранного дилатометра.

По мере увеличения температуры также увеличивается и КЛТР материалов, следовательно, усиливается влияние температурных флуктуаций на результат измерений. При температуре 300 К шум показаний емкостного датчика оказывается значительно больше, чем при температуре кипения жидкого гелия, поэтому нам не удалось добиться удовлетворительных результатов при постепенной развертке электрического поля, однако ошибка показаний емкостного датчика является накопительной по времени, поэтому можно проводить измерения обратного пьезоэлектрического эффекта при кратковременном резком изменении приложенного электрического поля. В результате этого накопленная ошибка окажется пренебрежимо малой.

Нами также были проведены измерения обратного пьезоэлектрического эффекта на монокристалле $\mathrm{Bi}_{12} \mathrm{GeO}_{20}$ при температуре 300 К. Экспериментальные значения пьезоэлектрического модуля $\mathrm{d}_{14}$ в этом монокристалле приведены в работе [11]. Авторами [12] исследовано изменение константы $\mathrm{d}_{14}$ при проявлении эффектов фотопроводимости, которые лежат в пределах от $1,5 \cdot 10^{-11}$ Кл/Н до $4,2 \cdot 10^{-11}$ Кл/Н. Значения относительных 
деформаций кристалла при продольном пьезоэффекте [13] в направлении оси 3-го порядка, для приведенных величин пьезомодулей $\mathrm{d}_{14}$ и при электрических полях $10 \kappa \mathrm{B} / \mathrm{cm}$ лежат в пределах $(0,8 \div 2,4) \cdot 10^{-5}$. Абсолютные деформации образца толщиной 1 мм составляют 8-24 нм. В эксперименте было получено значение 13 нм.

\section{ЗАКЛЮЧЕНИЕ}

Разработан и собран емкостный дилатометр для работы на базе измерительной установки PPMS QD, позволяющий проводить измерения магнитострикции, коэффициента линейного температурного расширения и обратного пьезоэлектрического эффекта, последнее было реализовано впервые на емкостном дилатометре. Дилатометр способен работать в интервале температур от 1.85 до 350 К, магнитных полей до 90 кЭ и электрических напряжений приложенных к образцу до 1 кВ.

Впервые измерен обратный пьезоэлектрический эффект в алюмоборате гольмия $\mathrm{HoAl}_{3}\left(\mathrm{BO}_{3}\right)_{4}$ при температуре 4.2 К. Значение пьезомодуля $d_{11}$ составляет $2.66 \cdot 10^{-10} \mathrm{~cm} / \mathrm{B}$. Полученные данные позволят провести оценочные расчеты магнитоэлектрического эффекта, вызванного взаимодействием упругих деформаций (магнитострикция и пьезоэлектрический эффект), что поможет объяснить природу магнитоэлектрического эффекта на макроскопическом уровне в семействе редкоземельных парамагнитных боратов.

Работа выполнена при финансовой поддержке Российского фонда фундаментальных исследований, Правительства Красноярского края, Красноярского краевого фонда поддержки научной и научно-технической деятельности» в рамках научного проекта № 16-48-243040, а также при поддержке Российского фонда фундаментальных исследований, проект №16-38-00245. 
1. Kuchler R., Stingl C., Gegenwart P. // Rev. Sci. Instrum. 2016 V. 87. P. 073903.

2. Shang M., Zhang C., Zhang T., Yuan L., Ge L., Yuan H., Feng S. // Appl. Phys. Lett. 2013 V. 102. P. 062903.

3. Ramesh R., Spaldin N. A., Nature Mater. 2007. V. 6. P. 21.

4. Kitagawa Y., Hiraoka Y., Honda T., Ishikura T., Nakamura H., Kimura T. // Nature Mater. 2010 V. 9. P. 797

5. Schmiedeshoff G. M., Lounsbury A. W., Luna D. J., Tracy S. J., Schramm A. J., Tozer S. W., Correa V. F., Hannahs S. T., Murphy T. P., Palm E. C., Lacerda A. H., Bud'ko S. L., Canfield P. C., Smith J. L., Lashley J. C., Cooley J. C. // Rev. Sci. Instrum. 2006 V. 77. P. 123907

6. Утюж А. Н., Краснорусский В. Н. // ПТЭ. 2011. Н 6, С. 133-136.Utyuzh A. N., Krasnorusskii V. N. // Instrum. Exp. Tech. 2011. V. 54. N 6. P. 872-875.

7. Kuchler R., Bauer T., Brando M., Steglich F. // Rev. Sci. Instrum. 2012. V. 83. P. 095102.

8. Liang K.-C., Chaudhury R. P., Lorenz B., Sun Y.Y., Bezmaternykh L.N., Temerov V.L., Chu C.W. // Phys. Rev. B. 2011. V. 83. P. 180417(R).

9. Freydman A.L., Balaev A.D., Dubrovskiy A.A., Eremin E.V., Temerov V.L., Gudim I.A. // J. Appl. Phys. 2014. V. 115. P. 174103.

10. Бегунов А.И., Демидов А.А., Гудим И.А., Еремин Е.В. // Письма в ЖЭТФ. 2013. Т. 97 B. 9. C. 611-618.

Begunov A. I., Demidov A. A., Gudim I. A., Eremin E. V. // JETP Letters. 2013. V. 97. N 9, P. 528-534.

11. Александров К.С., Сорокин Б.П., Бурков С.И. Эффективные пьезоэлектрические кристаллы для акустоэлектроники, пьезотехники и сенсоров // Новосибирск: Издво СО РАН. 2007. Т. 1. 501с. ISBN 978-5-7692-0914-7. 
12. Чмырев В.И., Скориков В.М., Ермагов Г.А. // ФТТ. 1980. Т.22. Н10. С3164-3166.

13. Сиротин Ю.И., Шаскольская М.П. Основы кристаллофизики // М.: Наука. 1979. 640c. 


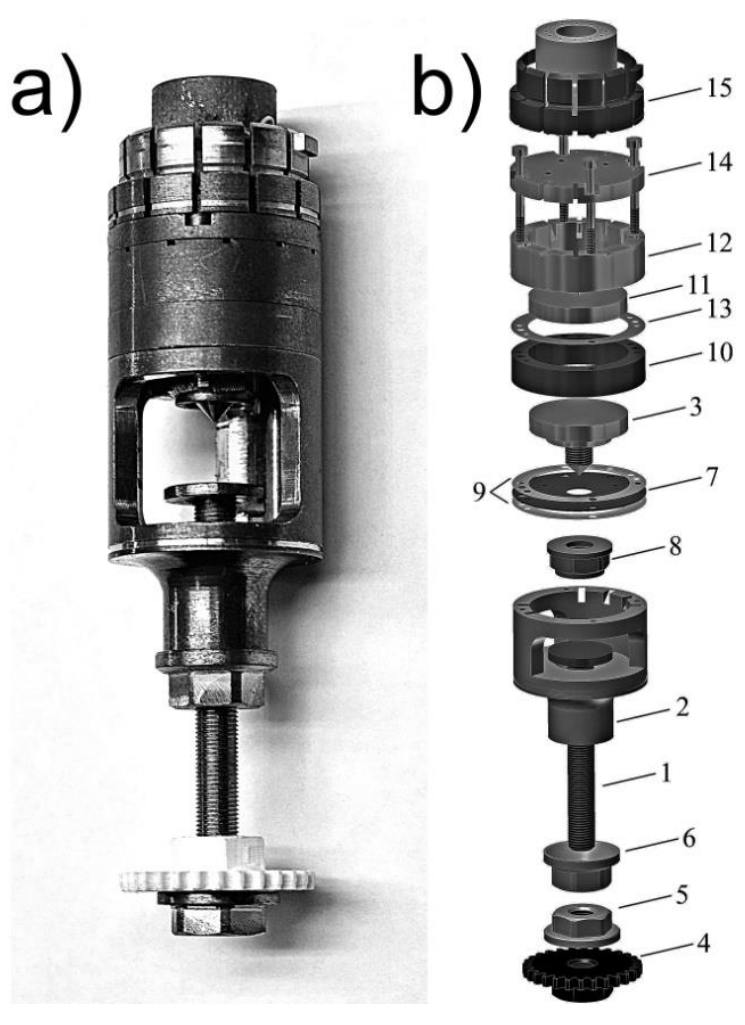

Рис. 1. Фрейдман. Емкостной дилатометр для измерения магнитострикции, пьезоэлектрического эффекта и коэффициента линейного температурного расширения. 


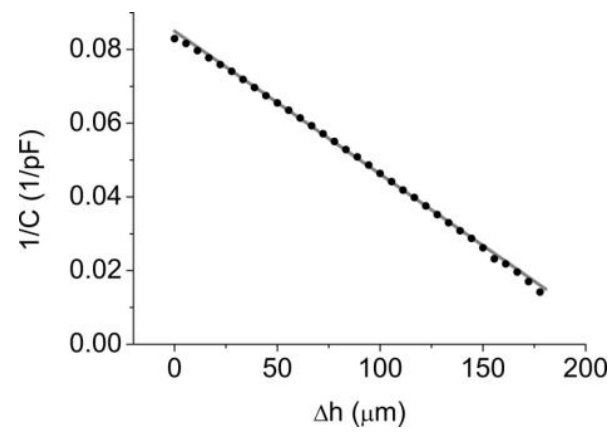

Рис. 2. Фрейдман. Емкостной дилатометр для измерения магнитострикции, пьезоэлектрического эффекта и коэффициента линейного температурного расширения. 

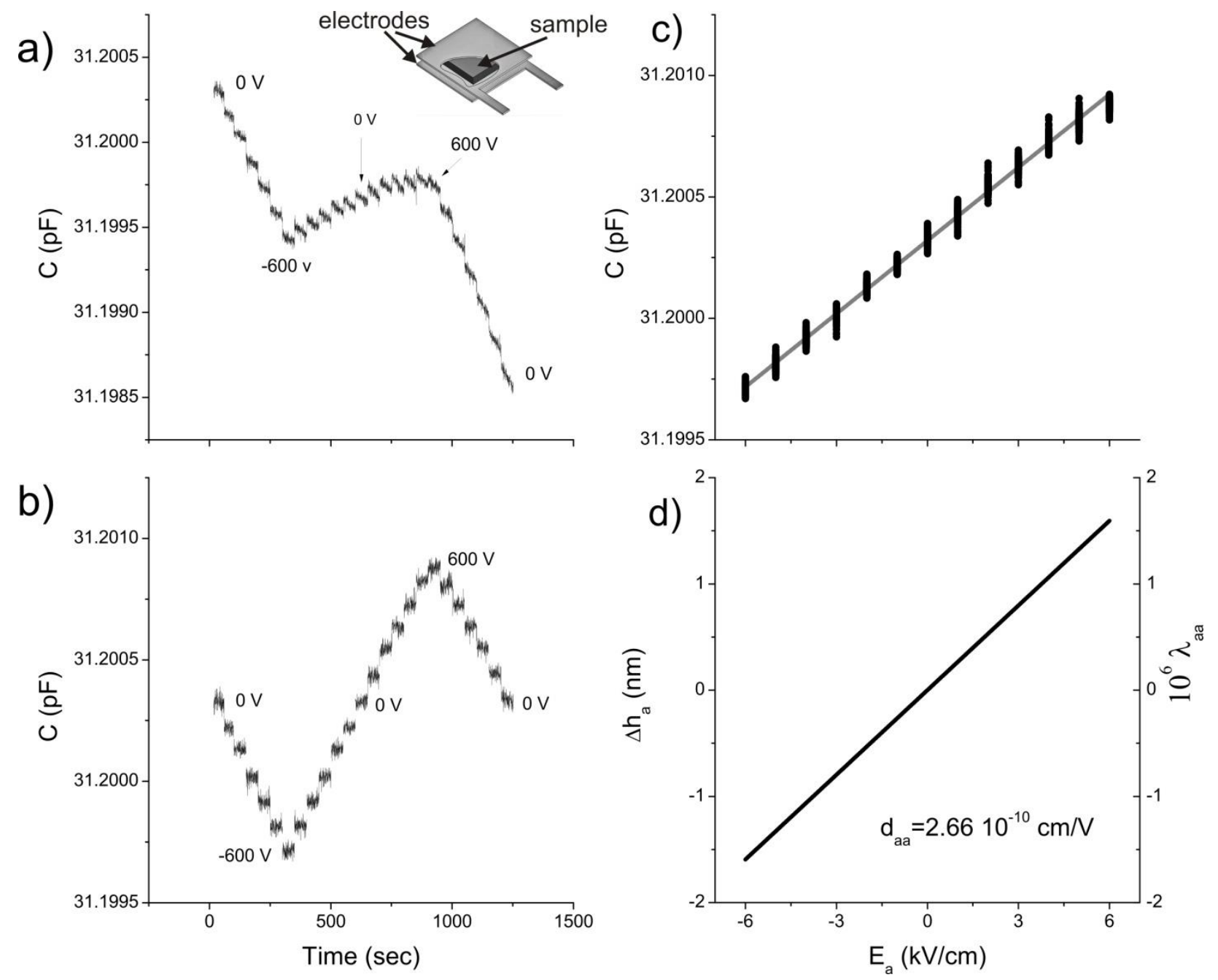

Рис. 3. Фрейдман. Емкостной дилатометр для измерения магнитострикции, пьезоэлектрического эффекта и коэффициента линейного температурного расширения. 
Рис. 1. Внешний вид измерительной дилатометрической ячейки (a) и ее состав (b). 1 столик образца, 2 - корпус измерительной ячейки, 3 - подвижная обкладка, 4 - зубчатое колесо, 5 - гайка зубчатого колеса, 6 - контргайка столика образца, 7 - мембрана, 8 - гайка подвижной обкладки, 9 - прокладка полиимидная, 10 - кольцо подвижной обкладки, 11 неподвижная обкладка, 12 - кольцо неподвижной обкладки, 13 - прокладка медная, 14 переходная пластина, 15 - электрический разъем.

Рис. 2. Зависимость обратной емкости измерительного конденсатора от смещения подвижной обкладки. Точками обозначены экспериментальные данные, а прямой линией линейная аппроксимация.

Рис. 3. Полученные экспериментальные данные пьезоэлектрического эффекта в $\mathrm{HoAl}_{3}\left(\mathrm{BO}_{3}\right)_{4}$ при температуре $T=4.2$ К. Зависимость емкости измерительного конденсатора от времени $(a, b)$ при ступенчатом изменении приложенного к образцу электрического поля, наблюдается экспериментальный дрейф емкости (a), (b) - данные после вычета временного дрейфа. Зависимость емкости измерительного конденсатора от приложенного к образцу электрического поля (с). Абсолютная $\Delta h_{\mathrm{a}}$ и относительная $\lambda_{\text {aа }}$ деформации монокристалла (d). На вставке к рисунку а показана схема подготовки образца. 